\title{
ON P-CLASS GROUPS AND THE FONTAINE-MAZUR CONJECTURE
}

\author{
JOCHEN GÄRTNER
}

\begin{abstract}
Answering a question by Stark, we show that for an infinite unramified pro- $p$-extension of a number field $k$, the $p$-class numbers of its finite subextensions tend to infinity. This is proven by means of a group-theoretical result on compact $p$-adic analytic groups. Furthermore, we provide an equivalent formulation of the FontaineMazur conjecture for $p$-extensions unramified outside a finite set of primes not containing any prime above $p$.
\end{abstract}

\section{Introduction}

The study of $p$-class numbers in extensions of number fields has a long and interesting history. Being closely related to deep questions in the realm of $p$-adic Galois representations, it is still among the central topics in algebraic number theory. In the classical case of $\mathbb{Z}_{p}$-extensions, the fundamental results of Iwasawa reveal a precise description of the growth behavior of $p$-class numbers. In particular, whether the size of the $p$-class groups stays bounded along a given $\mathbb{Z}_{p}$-extension depends on its $\lambda$ and $\mu$-invariants. While there do exist $\mathbb{Z}_{p}$-extensions with unbounded $p$-class numbers, Greenberg's conjecture predicts that they should always stay bounded for the cyclotomic $\mathbb{Z}_{p}$-extensions of totally real number fields.

Contrary to Iwasawa theory, which in general involves $p$-adic Lie extensions, i.e., extensions with $p$-adic analytic Galois groups, coming from algebraic geometry and being ramified above $p$, analogous questions are also of great interest in the situation of unramified or more generally tamely ramified (i.e., unramified outside a finite set of primes not containing any primes above $p$ ) pro- $p$-extensions. As shown by Golod and Šafarevič, there exist infinite unramified pro- $p$-extensions. However, the structure of their Galois groups is still very mysterious. One of the most important open questions in this context is the famous conjecture due to Fontaine and Mazur, stating that there exist no infinite tamely ramified pro- $p$-extensions with $p$-adic analytic Galois group (cf. [5], Conjecture 5a).

For unramified extensions, the Fontaine-Mazur conjecture is closely related to the following question asked by Stark (cf. [7]): Let $k^{\prime} \mid k$ be an infinite unramified pro-pextension of the number field $k$. Are the p-class numbers unbounded along the finite subextensions of $k^{\prime} \mid k$ ?

Applying results of Lubotzky and Mann characterizing $p$-adic analytic pro- $p$-groups by the finiteness of their Prüfer rank, Hajir explains that the Fontaine-Mazur conjecture would imply the following even stronger statement: the ranks of the $p$-class groups of the finite subextensions of $k^{\prime} \mid k$ tend to infinity (cf. [7]).

Received by the editors June 6, 2013. 
In this paper, we give an affirmative answer to Stark's question (cf. Theorem 7). The proof is of group-theoretical nature. We show that a compact $p$-adic analytic group $G$ either contains an open subgroup $H_{0}$ such that the abelianization of any open subgroup of $H_{0}$ is torsion free or the torsion parts of the abelianizations of open subgroups of $G$ have unbounded exponents (cf. Theorem 1). Together with the results of Lubotzky and Mann, this shows the following interesting dichotomy for an arbitrary infinite FAb pro-p-group: either it is non-analytic and hence the ranks of (the abelianizations) of its open subgroups are unbounded, or it is analytic and therefore by Theorem 1 the corresponding exponents necessarily grow to infinity.

In the second part of the paper, we consider the more general situation of tamely ramified extensions. It is shown how Theorem 1 yields an equivalent formulation of the aforementioned Fontaine-Mazur conjecture (cf. Conjecture 12 and Theorem 13). In fact, using results from class field theory, the conjecture can be equivalently stated in terms of boundedness of $p$-class numbers and local unramified extensions realized by a given tamely ramified $p$-adic Lie extension.

\section{Group-theoretical results}

Let $p$ be a prime number. For a profinite group $G$, by $\left(G_{i}\right)_{i \geq 1}$ we denote its lower $p$-central series, i.e.,

$$
G_{1}=G, G_{i+1}=G_{i}^{p}\left[G_{i}, G\right] .
$$

By $G^{a b}=G /[G, G]$ we denote the abelianization of $G$. Finally, if $G$ is abelian, let $\operatorname{Tor}(G)$ be the torsion subgroup and $\exp (\operatorname{Tor}(G))$ its exponent.

Theorem 1. Let $G$ be a compact p-adic analytic group. Then exactly one of the following holds:

(i) There exists an open subgroup $H_{0} \subseteq G$ such that $H^{a b}$ is torsion free for all open subgroups $H \subseteq H_{0}$, or

(ii) For every open powerful pro-p-subgroup $U \subseteq G$,

$$
\lim _{k \rightarrow \infty} \exp \left(\operatorname{Tor}\left(\left(U_{k}\right)^{a b}\right)\right)=\infty \text {. }
$$

\section{Remark 2.}

(i) Theorem 1 is trivial in the case of a finite $p$-group $G$, which obviously satisfies the first condition.

(ii) The above result seems to be known to the experts, however we could not find it in the literature. It has kindly been pointed out to the author by Klopsch that the first condition is equivalent to the following: $G$ is virtually abelian, i.e., there exists an open abelian subgroup of $U \subseteq G$. In fact, assume $H_{0} \subseteq G$ is given as in condition (i) of Theorem 1. By passing to an open subgroup, we may assume that $H_{0}$ is a uniform pro-p-group (cf. [2], Corollary 8.34). However, since $H_{0}$ has torsion free abelianization, it follows that $H_{0}$ is itself abelian (e.g., see [2], Proposition 4.32). Conversely, it is clear that any virtually abelian compact $p$-adic analytic group satisfies (i). 
We need the following Lemma which follows as a special case from [3], Corollary 3.5:

Lemma 3. Let $G$ be a powerful pro-p group. Then for all $k, k^{\prime} \geq 1, i, j \geq 0$

$$
\left[G_{k}, G_{k^{\prime}}\right]^{p^{i+j}}=\left[\left(G_{k}\right)^{p^{i}},\left(G_{k^{\prime}}\right)^{p^{j}}\right]=\left[G_{k+i}, G_{k^{\prime}+j}\right] .
$$

Proof of Theorem 1. First assume that (i) holds. By [2], Corollary 8.34, there exists an open uniform pro- $p$ subgroup $U \subseteq H_{0}$. By assumption $\operatorname{Tor}\left(\left(U_{l}\right)^{a b}\right)$ is trivial for all $l \geq 1$, hence (ii) does not hold.

Conversely assume that we are not in case (i). Let $U \subseteq G$ be an open powerful pro- $p$ subgroup. By [2], Theorem 4.2 we may assume that $U$ is uniform. Suppose that

$$
\exp \left(\operatorname{Tor}\left(\left(U_{k}\right)^{a b}\right)\right) \mid p^{n}
$$

for all $k \geq 1$ and some $n \geq 0$. By assumption, there exists an open subgroup $H \subseteq U_{n+1}$ of $U_{n+1}$ such that $\operatorname{Tor}\left(H^{a b}\right)$ is non-trivial. Let $g \in H$ such that $g[H, H] \in \operatorname{Tor}\left(H^{a b}\right)$ is not the identity. Furthermore, let $r \geq n+1$ be maximal such that $g \in U_{r}$, i.e., $g \in U_{r} \backslash U_{r+1}$. Since

$$
g^{p^{n}} \in[U, U]
$$

for $t=r-(n+1)$ it follows by Lemma 3 that

$$
g^{p^{n+2 t}} \in[H, H]^{p^{2 t}} \subseteq\left[U_{n+1}, U_{n+1}\right]^{p^{2 t}}=\left[\left(U_{n+1}\right)^{p^{t}},\left(U_{n+1}\right)^{p^{t}}\right]=\left[U_{r}, U_{r}\right],
$$

i.e. $g\left[U_{r}, U_{r}\right] \in \operatorname{Tor}\left(\left(U_{r}\right)^{a b}\right)$. However, this implies that

$$
g^{p^{n}} \in\left[U_{r}, U_{r}\right] \subseteq U_{2 r} .
$$

On the other hand, since $U$ is uniform, the map $x \mapsto x^{p}$ induces an isomorphism

$$
U_{i} / U_{i+1} \stackrel{\sim}{\longrightarrow} U_{i+1} / U_{i+2}
$$

for all $i \geq 1$ and therefore $g^{p^{n}} \in U_{r+n} \backslash U_{r+n+1}$. It follows that $r+n \geq 2 r$ which yields a contradiction. ${ }^{1}$

We now want to apply this result to the study of the subgroup growth in FAb pro-p groups.

Definition 4. A finitely generated pro- $p$ group $G$ is called $F A b$ if for every open subgroup $H \subseteq G$ the abelianization $H^{a b}=H /[H, H]$ is finite. ${ }^{2}$

As an immediate consequence of Theorem 1 we obtain the following:

Corollary 5. Let $G$ be an infinite FAb p-adic analytic pro-p group. Then

$$
\exp \left(\operatorname{Tor}\left(H^{a b}\right)\right) \longrightarrow \infty
$$

if $H$ runs through the open normal subgroups of $G$.

\footnotetext{
${ }^{1}$ This conclusion is inspired by an argument given by Labute in the proof of [11], Lemma 3.2.

${ }^{2}$ In the literature, the $F A b$ property is sometimes also denoted FIFA.
} 
For arbitrary (i.e., not necessarily $p$-adic analytic) FAb pro- $p$ groups we will prove the following:

Theorem 6. Let $G$ be an infinite FAb pro-p group. Then

$$
\# H^{a b} \longrightarrow \infty
$$

if $H$ runs through the open normal subgroups of $G$.

Recall that for a pro-p group $G$ the (Prüfer) rank $\operatorname{rk}(G)$ is defined as

$$
\operatorname{rk}(G)=\limsup \left\{\operatorname{dim}_{\mathbb{F}_{p}} H^{1}\left(H, \mathbb{F}_{p}\right) \mid H \leq G \text { closed subgroup }\right\} .
$$

By results of Lubotzky and Mann (cf. [12]),

$$
\begin{aligned}
\operatorname{rk}(G) & =\limsup \left\{\operatorname{dim}_{\mathbb{F}_{p}} H^{1}\left(H, \mathbb{F}_{p}\right) \mid H \leq G \text { open subgroup }\right\} \\
& =\limsup \left\{\operatorname{dim}_{\mathbb{F}_{p}} H^{1}\left(H, \mathbb{F}_{p}\right) \mid H \unlhd G \text { open normal subgroup }\right\} .
\end{aligned}
$$

We have the following characterization of $p$-adic analytic pro- $p$ groups, cf. [12], Th.A:

Lubotzky-Mann: A pro-p group $G$ is p-adic analytic if and only if $\operatorname{rk}(G)<\infty$.

We can now prove Theorem 6 .

Proof of Theorem 6. If $\exp \left(H^{a b}\right)$ is unbounded for $H$ running over the open normal subgroups of $G$, we are done. Hence assume $\exp \left(H^{a b}\right) \mid p^{n}$ for some $n \in \mathbb{N}$ and all open normal subgroups $H$. By Corollary 5 we deduce that $G$ is not $p$-adic analytic. Now the above result due to Lubotzky and Mann yields $\operatorname{rk}(G)=\infty$ which in particular implies $\# H^{a b} \rightarrow \infty$ and concludes the proof.

\section{Number theoretical results}

Let $k$ be a number field. We denote by $h_{k}(p)$ the $p$-class number, i.e.,

$$
h_{k}(p)=\# \mathrm{Cl}(k)(p) .
$$

From Theorem 6, we deduce the following result giving an affirmative answer to Stark's question (cf. [7]):

Theorem 7. Let $k$ be a number field and $k^{\prime} \mid k$ be an infinite unramified p-extension. Then $h_{K}(p) \rightarrow \infty$ if $K$ runs through the finite normal subextensions of $k^{\prime} \mid k$.

Proof. Since the Galois group $\operatorname{Gal}\left(k^{\prime} \mid k\right)$ is FAb, this follows immediately by Theorem 6 and the class field theory.

\section{Remark 8 .}

(i) An analogous result holds under the weaker assumption that the extension $k^{\prime} \mid k$ is ramified at a finite set of primes $S$ of $k$ not containing any prime above $p$. As in the unramified case, the Galois group $\operatorname{Gal}\left(k^{\prime} \mid k\right)$ is FAb and hence 
the statement carries over if for a finite subextension $K$ of $k^{\prime} \mid k$ the ideal class group $\mathrm{Cl}(K)(p)$ is replaced by the $p$-part of the ray class group

$$
\lim _{\mathfrak{m} \in \operatorname{supp}(S(K))} I_{K} / I_{K}^{\mathfrak{m}} K^{\times}
$$

Here $S(K)$ denotes the set of primes of $K$ lying above $S$ (see below for a review of the notation from global class field theory which is used here).

(ii) As mentioned in the Introduction, the above statement would also follow as a consequence of the Fontaine-Mazur conjecture. In fact, even more is true: if $k^{\prime} \mid k$ is an infinite unramified $p$-extension, the conjecture predicts that $\operatorname{Gal}\left(k^{\prime} \mid k\right)$ is not $p$-adic analytic and hence the $p$-class ranks of the finite normal subextensions $K \mid k$ are unbounded, i.e.,

$$
\operatorname{dim}_{\mathbb{F}_{p}} \mathrm{Cl}(K) / p \longrightarrow \infty .
$$

Conversely, this growth behavior implies the Fontaine-Mazur conjecture in the case where $k^{\prime} \mid k$ is the $p$-class field tower of $k$ (cf. [7]). The only explicit examples of infinite unramified pro- $p$-extensions we have at hand use (variations) of the Golod-Šafarevič inequality. In particular, their Galois groups are not $p$-adic analytic. However, e.g., it is not known whether for a given number field with infinite $p$-class field tower $k^{\prime} \mid k$, there exists a finite subextension $K \mid k$ such that $\operatorname{Gal}\left(k^{\prime} \mid K\right)$ satisfies the Golod-Safarevič inequality (cf. [7, 8]). Against this background, it seems useful to have the unconditional statement in Theorem 7. Moreover, Corollary 5 gives rise to an equivalent formulation of the Fontaine-Mazur conjecture (cf. Conjecture 12 and Theorem 13 below).

(iii) It is interesting to compare Theorem 7 to the behavior of $p$-class numbers in wildly ramified $p$-adic Lie extensions that naturally arise in the Iwasawa theory, such as $\mathbb{Z}_{p}$-extensions. If $k_{\infty} \mid k$ is the cyclotomic $\mathbb{Z}_{p}$-extension of a totally real number field $k$, Greenberg's conjecture [6] predicts that for the Iwasawa $\mu$ - and $\lambda$-invariants we have

$$
\mu\left(k_{\infty} \mid k\right)=\lambda\left(k_{\infty} \mid k\right)=0
$$

i.e., the size of the $p$-ideal class groups stays bounded along $k_{\infty} \mid k$. On the other hand, it has been shown by Iwasawa that the $\mu$-invariant can also become arbitrarily large for non-cyclotomic $\mathbb{Z}_{p}$-extensions.

In order to study the relation between our group-theoretical statements and the Fontaine-Mazur Conjecture, we need some results from the class field theory.

Let $S$ be a set of primes of the number field $k$. We denote by $k_{S}\left(\operatorname{resp} . k_{S}(p)\right)$ the maximal extension (resp. maximal pro-p-extension) of $k$ unramified outside $S$ and set

$$
G_{k, S}:=\operatorname{Gal}\left(k_{S} \mid k\right), \quad G_{k, S}(p):=\operatorname{Gal}\left(k_{S}(p) \mid k\right) .
$$

In this section, we recall some facts from the class field theory to estimate the exponent $\exp \left(G_{k, S}(p)^{a b}\right)$ where $S$ is a finite set of finite primes of $k$ not containing any primes above $p$. 
Let $I_{k}$ denote the idèle class group of $k$. Recall that a modulus $\mathfrak{m}$ of $k$ is a formal product

$$
\mathfrak{m}=\prod_{\mathfrak{p}} \mathfrak{p}^{n_{\mathfrak{p}}}
$$

where $\mathfrak{p}$ runs through all primes of $k$ and $n_{\mathfrak{p}} \geq 0$ is an integer for all $\mathfrak{p}$ such that

(i) $n_{\mathfrak{p}}=0$ for all but finitely many $\mathfrak{p}$;

(ii) $n_{\mathfrak{p}} \in\{0,1\}$ if $\mathfrak{p}$ is an infinite prime.

By $I_{k}^{\mathfrak{m}} \subset I_{k}$ we denote the subgroup of all idèles $\alpha=\left(\alpha_{\mathfrak{p}}\right)_{\mathfrak{p}}$ such that

(i) $\alpha_{\mathfrak{p}} \in U_{\mathfrak{p}}^{n_{\mathfrak{p}}}$ for all finite primes $\mathfrak{p}$ where $U_{\mathfrak{p}}^{n_{\mathfrak{p}}}$ denotes the $n_{\mathfrak{p}}$-th higher unit group and we set $U_{\mathfrak{p}}^{n_{\mathfrak{p}}}=U_{\mathfrak{p}}$ if $n_{\mathfrak{p}}=0$.

(ii) $\alpha_{\mathfrak{p}} \in \mathbb{R}_{+}^{\times}$if $\mathfrak{p}$ is a real prime such that $n_{\mathfrak{p}}=1$.

By global class field theory, the quotient $I_{k} / I_{k}^{\mathfrak{m}} k^{\times}$is canonically isomorphic to the Galois group of the ray class field of $k$ modulo $\mathfrak{m}$. We denote by $\operatorname{supp}(S)$ the set of all moduli $\mathfrak{m}=\prod_{\mathfrak{p}} \mathfrak{p}^{n_{\mathfrak{p}}}$ of $k$ such that $n_{\mathfrak{p}}=0$ for all $\mathfrak{p} \notin S$. By the Existence Theorem of global class field theory, we have an isomorphism

$$
G_{k, S}^{a b} \stackrel{\sim}{\longrightarrow} \lim _{\mathfrak{m} \in \operatorname{supp}(S)} I_{k} / I_{k}^{\mathfrak{m}} k^{\times}
$$

Definition 9. Let $S$ be a finite set of finite primes of $k$ not containing any prime above $p$. We set

$$
e(S):=\max _{\mathfrak{p} \in S}\left\{v_{p}(N(\mathfrak{p})-1)\right\}
$$

Proposition 10. Let $S$ be a finite set of primes of $k$ not containing any prime above $p$. Then

$$
\exp \left(G_{k, S}(p)^{a b}\right) \leq p^{e(S)} \cdot \exp \left(\mathrm{Cl}_{k}(p)\right)
$$

Proof. Let $\mathfrak{m} \in \operatorname{supp}(S)$ and consider the exact sequence of abelian groups

$$
\prod_{\mathfrak{p} \in S} U_{\mathfrak{p}} \longrightarrow I_{k} / I_{k}^{\mathfrak{m}} k^{\times} \longrightarrow I_{k} / I_{k}^{1} k^{\times} \longrightarrow 1
$$

Here $I_{k}^{1}=\prod_{\mathfrak{p} \in S_{\infty}} k_{\mathfrak{p}}^{\times} \times \prod_{\mathfrak{p} \notin S_{\infty}} U_{\mathfrak{p}}$, where $S_{\infty}$ denotes the set of infinite primes of $k$, and we have a canonical isomorphism

$$
I_{k} / I_{k}^{1} k^{\times} \cong \mathrm{Cl}_{k} .
$$

Furthermore,

$$
U_{\mathfrak{p}} \cong \mu_{N(\mathfrak{p})-1} \times U_{\mathfrak{p}}^{1}
$$


and $U_{\mathfrak{p}}^{1}$ is a $\mathbb{Z}_{l_{\mathfrak{p}}}$-module where $l_{\mathfrak{p}} \neq p$ is the residue characteristic of the prime $\mathfrak{p}$. Hence the claim follows by taking pro- $p$ quotients and passing to the projective limit over all $\mathfrak{m} \in \operatorname{supp}(S)$.

\section{On the Fontaine-Mazur conjecture}

Let $k$ be a number field, $\bar{k}$ its algebraic closure. A continuous irreducible $p$-adic representation

$$
\rho: \operatorname{Gal}(\bar{k} \mid k) \longrightarrow \mathrm{GL}_{n}\left(\mathbb{Q}_{p}\right)
$$

is called geometric, if it is unramified outside a finite set of primes of $k$ and its restriction $\rho_{\mid G_{\mathfrak{p}}}$ to the decomposition group $G_{\mathfrak{p}}$ of $\mathfrak{p}$ is potentially semi-stable for all primes $\mathfrak{p}$ of $k$ dividing $p .{ }^{3}$ In [5], Fontaine and Mazur make the following fundamental conjecture: $\rho$ is geometric if and only if it comes from algebraic geometry, i.e., it arises from the Galois action on an étale cohomology group $H_{e t}^{i}\left(V_{\bar{k}}, \mathbb{Q}_{p}(j)\right)$ of a smooth projective variety $V$ over $k$. It has been proven in the $\mathrm{GL}_{2}$-case (under some further assumptions) by Kisin (cf. [9]). Under the assumption of further conjectures by Tate and Grothendieck-Serre, it implies the following prediction about tamely ramified representations (cf. [10] for the details):

Conjecture 11 (Fontaine-Mazur [5], Conjecture 5a). Let $k$ be a number field and

$$
\rho: \operatorname{Gal}(\bar{k} \mid k) \longrightarrow \mathrm{GL}_{n}\left(\mathbb{Q}_{p}\right)
$$

a continuous irreducible presentation which is unramified outside a finite set $S$ of primes of $k$ not containing any prime above $p$. Then $\rho$ factors through a finite quotient of $\operatorname{Gal}(\bar{k} \mid k)$.

Equivalently, the conjecture states that there exist no infinite tamely ramified $p$ adic Lie extensions. It has been proven in special situations, cf. [1, 7, 14] (for the case $S=\varnothing$ ) and [13]. In this section, using Corollary 5, we give the following equivalent formulation:

Conjecture 12. Let $k$ be a number field and $k^{\prime} \mid k$ a p-adic Lie extension unramified outside a finite set of primes $S$ of $k$ not containing any prime above $p$. Then the following holds:

(i) There exists a number $n \in \mathbb{N}$ such that $\exp (\mathrm{Cl}(K)(p)) \mid p^{n}$ for any finite normal subextension $K \mid k$ inside $k^{\prime}$.

(ii) For $\mathfrak{p} \in S$ and a prime $\mathfrak{P}$ of $k^{\prime}$ lying above $\mathfrak{p}$, the local extension $k_{\mathfrak{P}}^{\prime} \mid k_{\mathfrak{p}}$ does not contain the maximal unramified pro-p-extension of $k_{\mathfrak{p}}$.

Theorem 13. Conjectures 11 and 12 are equivalent.

Proof. For a number field $k$, we set $S_{p, k}:=\{\mathfrak{p}$ prime of $k$ such that $\mathfrak{p} \mid p\}$. Assume that Conjecture 11 holds. Then any $p$-adic Lie extension $k^{\prime} \mid k$ unramified outside a finite set of primes $S$ with $S \cap S_{p, k}=\varnothing$ is finite, hence Conjecture 12 holds.

\footnotetext{
${ }^{3}$ The notion of potentially semi-stable $p$-adic representations is due to Fontaine, cf. [4].
} 
Now let $k^{\prime} \mid k$ be a $p$-adic Lie extension unramified outside $S$ with $S \cap S_{p, k}=\varnothing$ and satisfying the conditions (i) and (ii) of Conjecture 12. It remains to show that $k^{\prime} \mid k$ is finite. Set $G=\operatorname{Gal}\left(k^{\prime} \mid k\right)$. By passing to a finite normal subextension, we may assume that $G$ is a $p$-adic analytic pro- $p$ group. Since no prime above $p$ is ramified in $k^{\prime} \mid k$, it follows that $G$ is FAb. For a prime $\mathfrak{p} \in S$, choose a prime $\mathfrak{P} \mid \mathfrak{p}$ of $k^{\prime}$ and let $\left(k_{\mathfrak{P}}^{\prime}\right)^{n r} \mid k_{\mathfrak{p}}$ be the maximal unramified extension inside $k_{\mathfrak{p}}^{\prime} \mid k_{\mathfrak{p}}$. By condition (ii), the extension $\left(k_{\mathfrak{P}}^{\prime}\right)^{n r} \mid k_{\mathfrak{p}}$ is finite and we define $f_{\mathfrak{p}}$ by

$$
p^{f_{\mathfrak{p}}}=\left[\left(k_{\mathfrak{P}}^{\prime}\right)^{n r}: k_{\mathfrak{p}}\right]
$$

Set

$$
f(S):=\max _{\mathfrak{p} \in S}\left\{f_{\mathfrak{p}}\right\}
$$

Let $K \mid k$ be a finite normal subextension of $k^{\prime} \mid k$ and $\mathfrak{p}_{K}$ a prime of $K$ lying above $\mathfrak{p}$. Then

$$
v_{p}\left(N\left(\mathfrak{p}_{K}-1\right) \leq v_{p}(N(\mathfrak{p})-1)+f_{\mathfrak{p}} \leq e(S)+f(S)\right.
$$

where as in Section 3 we have set $e(S)=\max _{\mathfrak{p} \in S}\left\{v_{p}(N(\mathfrak{p})-1)\right\}$. If $S_{K}$ denotes the set of all primes of $K$ lying above $S$, the maximal abelian subextension of $k^{\prime} \mid K$ is contained in $K_{S_{K}}(p)^{a b}$ and hence by Proposition 10 and condition (i) we have

$$
\exp \left(\operatorname{Gal}\left(k^{\prime} \mid K\right)^{a b}\right) \leq \exp \left(G_{K, S_{K}}(p)^{a b}\right) \leq p^{e(S)+f(S)} \cdot p^{n} .
$$

By Corollary 5 we conclude that $G$ is finite.

\section{Acknowledgment}

The author would like to thank Kay Wingberg for valuable suggestions and comments.

\section{References}

[1] N. Boston, Some cases of the Fontaine-Mazur conjecture II, J. Number Theory 75 (1999), 161-169.

[2] J. Dixon, M. du Sautoy, A. Mann and D. Segal, Analytic pro-p groups, 2nd edn., Cambridge Studies in Advanced Mathematics 61, Cambridge University Press (1999).

[3] G. Fernández-Alcober, J. González-Sánchez and A. Jaikin-Zapirain, Omega subgroups of pro-p groups, Israel J. Math. 166 (2008), 393-412.

[4] J.-M. Fontaine, Répresentations p-adiques semi-stables, Astérisque $\mathbf{2 2 3}$ (1994, Périodes $p$-adiques (Bures-sur-Yvette, 1988)) 113-184.

[5] J.-M. Fontaine and B. Mazur, Geometric Galois representations, in 'Elliptic curves, modular forms \& Fermat's Last Theorem' (J. Coates and S. Yau, eds), 41-78, International Press, Boston (1995).

[6] R. Greenberg, On the structure of certain Galois groups, Invent. Math. 47 (1978), 85-99.

[7] F. Hajir, On the growth of p-class groups in p-class field towers, J. Algebra 188 (1997), 256-271.

[8] F. Hajir and C. Maire, Unramified subextensions of Ray class field towers, J. Algebra 249 (2002), 528-543.

[9] M. Kisin, Overconvergent modular forms and the Fontaine-Mazur conjecture, Invent. Math. 153(2) (2003), 373-454.

[10] M. Kisin and S. Wortmann, A note on Artin motives, Math. Res. Lett. 10(2-3) (2003), 275-389.

[11] J. Labute, Linking numbers and the tame Fontaine-Mazur conjecture, Annales mathématiques du Québec, 1-11, Springer International Publishing (2014). Doi: 10.1007/s40316-014-0012-4. 
[12] A. Lubotzky and A. Mann, Powerful p-groups. II: p-adic analytic groups, J. Algebra 105 (1987), $506-515$.

[13] C. Maire, Some new evidence for the Fontaine-Mazur conjecture, Math. Res. Lett. 14(4) (2007), $673-680$.

[14] K. Wingberg, On the Fontaine-Mazur conjecture for CM-fields, Compos. Math. 131(3) (2002), $341-354$.

Universitt Heidelberg, Mathematisches Institut, Im Neuenheimer Feld 288, 69120 Heidelberg, Germany

E-mail address: gaertner@mathi.uni-heidelberg.de 
